\title{
European Surveillance of Legionnaires' Disease
}

\section{Birgitta de Jong and Lara Payne Hallström*}

European Centre for Disease Prevention and Control (ECDC), Solna, Sweden

*lara.payne@ecdc.europa.eu

DOI: https://doi.org/10.21775/cimb.042.081

\begin{abstract}
Legionnaires' disease and Pontiac fever are both diseases with important public health implications and require prompt and thorough responses to outbreaks for future prevention. Effective methods for defining, diagnosing, reporting and responding to legionellosis outbreaks ideally should be standardized across countries. Therefore, the European Union surveillance methods for countering Legionnaires' disease is a useful model especially for travel-associated Legionnaires' disease (TALD) cases which are on the rise. Multi-country surveillance in the EU and European Economic Area (EEA) has evolved since the first organizational efforts in the 1980's to the currently responsible, European Legionnaires' Disease Surveillance Network (ELDSNet). This chapter outlines the practices of the EU surveillance of Legionnaires' disease including their schemata, definitions, responsibilities of participating members, methods and the results of the data collected since the program's inception. Lastly, improvements must still be made as the incidence of Legionnaires'
\end{abstract}


disease in the EU is likely underestimated due to underreporting and/or underdiagnosis. Nearly $70 \%$ of cases are reported from only four countries, France, Germany, Italy and Spain, which represent $50 \%$ of the EU population.

\section{Introduction}

Legionnaires' disease is a statutorily notifiable disease in all EU and EEA countries. However, the case definitions across different countries varies. In some countries legionellosis, in general, is a notifiable disease which includes cases of both Legionnaires' disease and Pontiac fever. However, in other countries only Legionnaires' disease is a notifiable disease. There are also discrepancies between countries in the laboratory methods used. A case definition that includes both epidemiological and microbiological criteria is therefore essential when performing surveillance. Such a definition should accomplish the following:

- Set standards for defining cases;

- Compare data between countries;

- Verify the diagnosis and share laboratory results;

- Facilitate international investigations and collaborations;

- Develop best practice within and between countries.

For EU surveillance, a case definition for Legionnaires' disease has been agreed on [See Box]. Since this case definition only deals with cases that have clinical criteria of pneumonia, all cases of Pontiac fever are excluded from the EU surveillance of Legionella infections.

Multi-country surveillance of Legionnaires' disease within Europe has existed since the mid-1980s. Since the establishment of the European Centre for Disease Prevention and Control (ECDC) in 2005, surveillance of Legionnaires' disease at the EU-level is carried out by ECDC. One of the ECDC's objectives is to strengthen the surveillance of communicable 


\section{EU case definition of Legionnaires' disease [\#Commission}

Implementing Decision 2012/506/EU of 8 August 2012 amending Decision 2002/253/EC laying down case definitions for reporting communicable diseases to the Community network under Decision No 2119/98/EC of the European Parliament and of the Council (2012).

\section{Clinical criteria}

Any person with pneumonia

\section{Laboratory criteria for case confirmation}

At least one of the following three:

$\square$ Isolation of Legionella spp. from respiratory secretions or any normally sterile site

$\square$ Detection of Legionella pneumophila antigen in urine

$\square$ Significant rise in specific antibody level to Legionella pneumophila serogroup 1 in paired serum samples.

\section{Laboratory criteria for a probable case}

At least one of the following four:

$\square$ Detection of Legionella pneumophila antigen in respiratory secretions or lung tissue, e.g. by DFA staining using monoclonalantibody-derived reagents

$\square$ Detection of Legionella spp. nucleic acid in respiratory secretions, lung tissue or any normally sterile site;

Significant rise in specific antibody level to Legionella pneumophila other than serogroup 1 or other Legionella spp. in paired serum samples

$\square$ Single high level of specific antibody to Legionella pneumophila serogroup 1 in serum.

\section{Case classification}

Probable case: Any person meeting the clinical criterion AND at least one laboratory criterion for a probable case Confirmed case: Any person meeting the clinical criterion AND at least one laboratory criterion for a confirmed case 
diseases in the EU and another is to provide guiding principles for response to public health threats related to communicable diseases at the EU level. For example, ECDC supports Member States by coordinating investigations of a large outbreaks that involve individuals from several different Member States or outbreaks occurring outside of the EU but affecting EU citizens. ECDC also collects annual surveillance data on Legionnaires' disease from the EU/EEA countries to analyse disease trends. To achieve these objectives of surveillance, a disease-specific network called the European Legionnaires' Disease Surveillance Network (ELDSNet) was established in 2010.

Representatives from the national authorities with responsibility for public health in each EU/EEA country nominate the official participatory members for the network. These members generally include one public health epidemiologist from the national public health institute or ministry of health and one microbiologist from the national or regional Legionella reference laboratory.

Prior to ECDC, a network called the European Working Group for Legionella Infections (EWGLI) was already established in 1986. The name, EWGLINET, was adopted in May 2002 in order to distinguish the surveillance from other activities carried out by EWGLI. From 1993 to the end of March 2010, EWGLI and EWGLINET was managed by the coordinating centre at the Health Protection Agency's Centre for Infections in London (formally the Communicable Disease Surveillance Centre of the Public Health Laboratory Service). From April 2010, running the network became the responsibility of ECDC and the network was renamed, ELDSNet. The prime aim of ELDSNet is to detect, control and prevent cases, clusters and outbreaks of Legionnaires' disease within EU/EEA countries. In addition, ELDSNet assists, where possible, with detection and response outside these countries. The network provides the means 
within the EU to share information and collaborate on Member State actions so that residents of European countries are increasingly protected from acquiring Legionnaires' disease linked to travel within their own countries or abroad.

\section{EU Surveillance}

The surveillance data are from two different schemes: the first scheme covers all cases reported annually from EU Member States and EEA countries with the following objectives:

- Monitor trends over time and to compare them across Member States

- Monitor the morbidity and mortality due to Legionnaires' disease and identify population groups at risk and in need of targeted preventive measures.

- Provide evidence-based data for public health decisions and actions by the EU and/or Member States level

The second near real-time reporting scheme covers travel-associated cases of Legionnaires' disease (TALD), including some reports from countries outside the EU/EEA. This second scheme primarily aims at identifying clusters of cases associated with accommodation sites that may otherwise not have been detected at the national level and enabling timely investigation and control measures at the implicated accommodation sites in order to prevent further cases of Legionnaires' disease.

\section{Data collection methods}

The collection of national data covering all reported cases annually is done through a dataset call in the beginning of the year. The appointed ELDSNet members in each European country are asked to electronically report to the European Surveillance System (TESSy) database all cases reported nationally in their country during the previous year following a 
strict protocol. About $20 \%$ of reported cases to the EU level since 2005 are travel-associated (domestic or international travel).

The travel-associated Legionnaires' disease surveillance scheme has distinct objectives. Particularly, ECDC receives reports of individual cases of TALD from TESSy on a daily basis in near real-time. The reporting country is generally the country where the case is diagnosed. Therefore, the reporting country can differ from the case's country of residence depending on the travel itinerary of the case. Case reports include age, sex, date of onset of disease, method of diagnosis and travel information for the places where the individual stayed within two to ten days prior to the onset of illness. Only cases who stayed at a commercial or public accommodation sites are reported as opposed to cases of Legionnaires' disease who stayed with relatives or friends. All network members transmit case information via a secure part of the ECDC web portal. Case reports received are reviewed, and the location is searched for other cases associated with the location at any time since 1987 when recording began. With complete and rapid reporting, the surveillance network can detect clusters of TALD in residents from two or more countries travelling to a single holiday destination or staying in the same commercial accommodation site. Receipt of the information leads to specific and timely action by the network countries in order to protect travellers staying at the indicated sites within EU. After receiving the report, each new case is classified as a single case or as part of a cluster in accordance with the definitions agreed upon by the network.

If the travel-associated infection is linked to countries outside the EU/EEA area, ECDC liaises with the national authorities either directly or through collaboration with the World Health Organization (WHO). This way, prevention of TALD is promoted outside the EU/EEA area. As at April 2018, TALD surveillance is the only indicator-based surveillance scheme operating at ECDC year-round as near real-time surveillance reporting. 
The daily surveillance of TALD at a European level supplements national level surveillance by identifying clusters of cases which may originate from different countries. These clusters otherwise may not have been detected at the national level. The rapid reporting of cases and clusters enable timely investigations and implementation of control measures at the implicated accommodation sites in order to prevent further infections.

\section{ELDSNet definitions of travel-associated Legionnaires' disease cases}

Single case

Case of Legionnaires' disease who in the two to ten days before onset of illness stayed at or visited a commercial accommodation site that has not been associated with any other cases of Legionnaires' disease, or case who stayed at an accommodation site linked to other cases with date of onset more than two years apart.

Cluster

Two or more cases who stayed at or visited the same commercial accommodation site in the two to ten days before onset of illness and whose onset is within the same two-year period.

A cluster can be additionally categorised as a:

* Rapidly evolving cluster: when three or more cases have onset of disease within a three-month period occurring in the six months preceding or following a cluster notification or update. This categorisation indicates a possible recent increase of exposure risk.

* Complex cluster: if a cluster involves cases that are associated with staying at one or more accommodation sites also being part of clusters, it is handled as a 'complex cluster'.

If any further cases associated with the cluster site occur more than two years after the last case, they will be reported as new single cases, although the country of infection will receive information on all previous cases linked to the accommodation site regardless of the time period elapsed. 
As at April 2018, 28 EU Member States, two EEA countries and two countries outside the EU/EEA area were contributing or receiving data on TALD cases. ELDSNet has contacts in 30 countries outside the EU/EEA area who are receiving data directly from ELDSNet.

\section{TALD notifications and investigations}

All notifications regarding single TALD cases and clusters aim to be sent out within one business day from the reception of the case report. Single cases are reported by ELDSNet to the country where the accommodation site is situated and to the reporting country. The hosting country is then expected to ensure that the notified site receives a checklist outlining good practices for minimising risk of Legionella infection. This checklist is found within a leaflet for managers running a tourist accommodation (ECDC Health Information). Many countries carry out environmental investigations for single cases, and the investigation assessment of these can be voluntarily submitted to ELDSNet.

Clusters are reported to the country where the accommodation site is located, the reporting country and all ELDSNet network members. The hosting country is expected to arrange for the accommodation site to be inspected by a competent institution authorised by the national authority such as a local or regional public health authority. This must be accomplished promptly as well as a risk assessment according to guidelines of the country or the European Technical guidelines (2017). This should be followed by an environmental investigation such as sampling of accommodation site water systems. The investigation informs the implementation of control measures to minimise any possible risk for Legionnaires' disease and support prevention. These actions should be reported to ELDSNet using the specific forms.

Form A should have information on whether or not an inspection and risk assessment have been carried out at the accommodation site. It should 
be returned to ECDC within two weeks of receipt of the cluster notification. Form A should state whether control measures are in progress and whether the hotel remains open or not. Form B should have information on the investigations and control measures that have been implemented at the accommodation site, including the results of sampling that took place. It should be returned to ECDC within six weeks of receipt of the cluster notification.

If these forms are not received from an EU/EEA country, or if it contains information that the accommodation site is not implementing any recommended control measures, then the site's name is published on the accommodations list on the public section of the ECDC website. The accommodation site list on the ECDC public website shows the current accommodation sites in the EU/EEA countries in which clusters of Legionnaires' disease have been identified, where there has been no complete assessment of the risk for Legionella infection and where ELDSNet believes there may be an increased risk to travellers.

Accommodation sites associated with clusters situated outside of the EU/ EEA area do not appear on the accommodation site list. Rather, these clusters are reported to those tour operators that are subscribed to receive specific summary reports from ELDSNet concerning the clusters. Subscribed tour operators are routinely informed of all clusters located in countries outside the EU/EEA area, because the ECDC does not require timely information on the control measures being implemented at these accommodation sites. In the event of a rapidly evolving cluster occurring inside EU/EEA area or worldwide, subscribed tour operators are informed. Tour operators on the subscriber list will be informed when an accommodation in an EU/EEA country is intended to be published or removed from the ECDC public website. Tour operators are also informed of any voluntarily completed form $B$ for other sites outside the EU/EEA area submitted by the national public health authorities. 


\section{Reported disease in the EU/EEA}

\section{All reported cases}

The number of reported cases in the EU/EEA countries has fluctuated during the last ten years (Figure 1). A sharp increase in the number of reported cases was seen in 2014 when a large outbreak affecting almost 400 people occurred in Portugal. However, even if this outbreak was not included in the figures, the year of 2014 had the highest reported number of cases (7022 cases). Since there were no changes in laboratory methods or reporting systems, this increase of cases remains unexplained. The high level of reported cases continued into 2015 with 7034 cases, and no major outbreaks were reported.

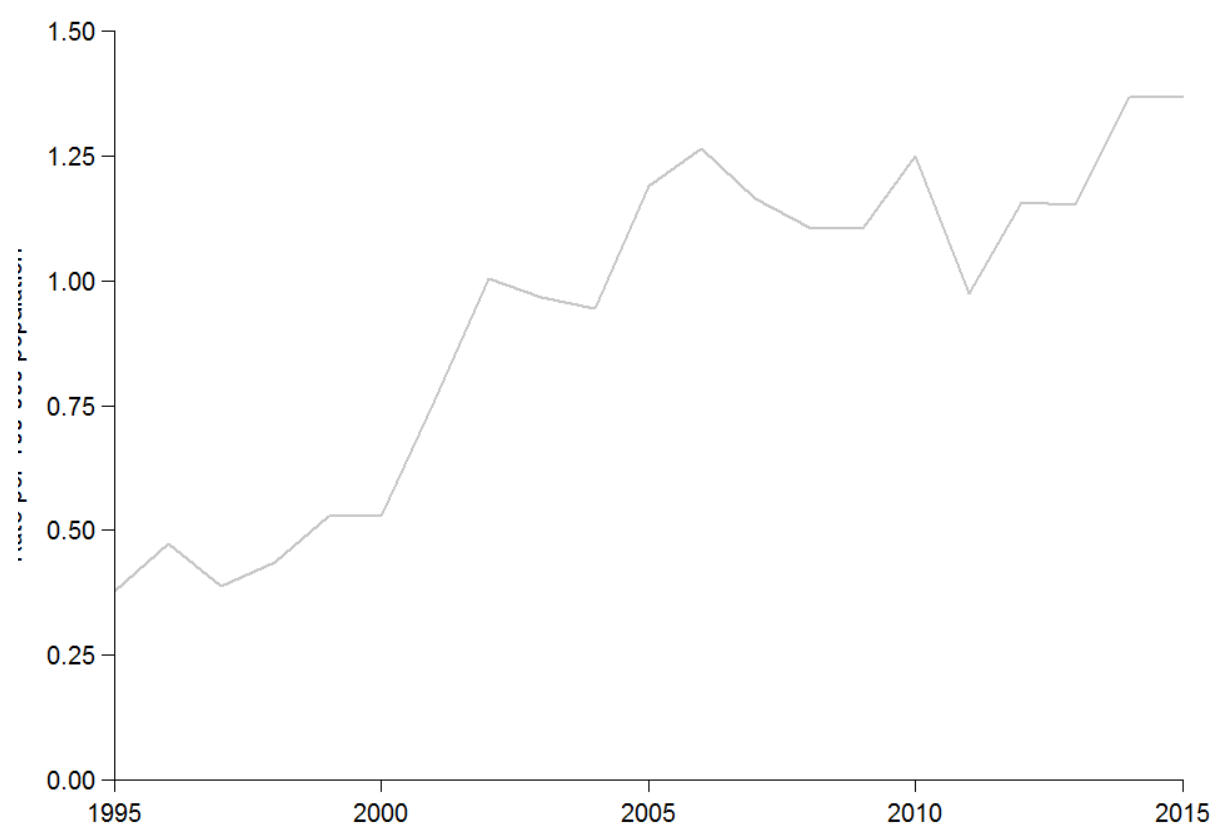

Figure 1. Notification rate of Legionnaires' disease in the EU/EEA* by year of reporting, 1995-2015. "EWGLINET member countries not belonging to the EU/EEA were excluded for 1995-2008. Source: https://ecdc.europa.eu/sites/portal/files/documents/Legionnares-disease-europe-2015.pdf 
The majority of cases are reported from countries in the Western part of Europe (Figure 2). Four countries, France, Germany, Italy and Spain usually report the vast majority of cases in the EU/EEA area. In 2015, these four countries they accounted for $69 \%$ of all cases. On the other hand, the 15 lowest reporting countries merely reported $3 \%$ of all cases. This shows how unevenly distributed the reported cases are among the EU/EEA countries.

There is a seasonal variation for Legionnaires' disease, and August is the month when most cases are reported to have onset of disease. Notification rates also increase with age. People older than 50 years of age accounted for $81 \%$ of cases with known age and sex in 2015. Legionnaires' disease is more common in males, with an overall male-to-

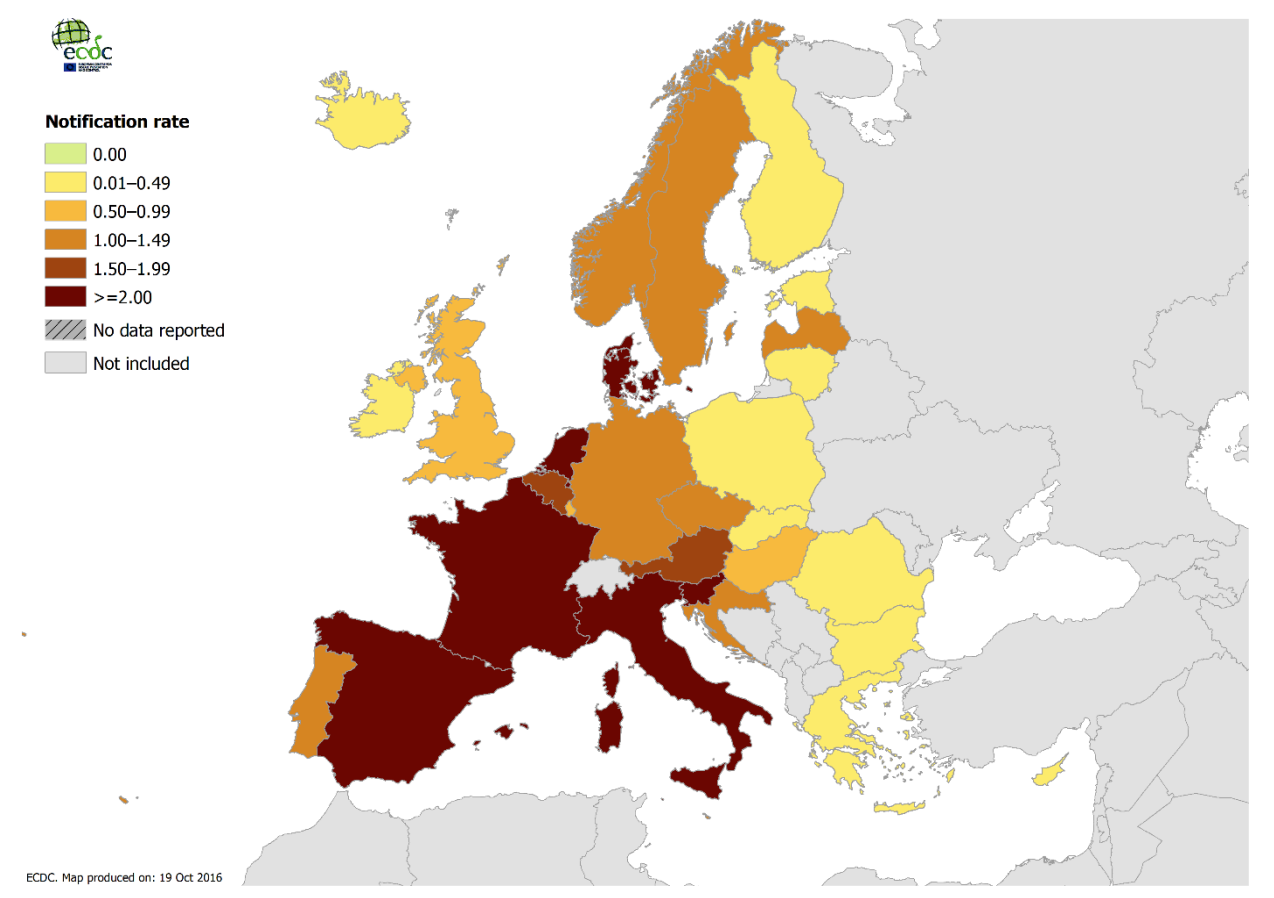

Figure 2. Notifications of Legionnaires' disease per 100 000, by reporting country, EU/EEA, 2015. Source: https://ecdc.europa.eu/sites/portal/files/documents/Legionnares-disease-europe-2015.pdf 
female ratio of $2.5-3.0$ to 1 . About $75 \%$ of the cases are reported as community-acquired and roughly $20 \%$ are travel associated. Healthcareassociated cases represents a considerable proportion of cases in older age groups, but in the total figures this setting represents less than $10 \%$.

The reported mortality rate of Legionnaires' disease in 2015 was 0.8 per million inhabitants, which is consistent with the rates recorded since 2008 which ranged from 0.7 to 0.9 per million inhabitants. Of 4893 cases with a known outcome, 396 were reported to have died giving a case-fatality ratio (CFR) of $8 \%$. Case fatality is higher for older age groups, both in males and females. In individuals above 50 years of age, case fatality is higher. A similar pattern is demonstrated for both males and females.

Almost $90 \%$ of cases are reported to have been diagnosed with a urinary antigen test, but there is a large variety in diagnostic tests used in the different EU countries. PCR tests are increasingly used, and in some countries more than $20 \%$ of cases are diagnosed with PCR.

\section{Travel-associated cases}

The number of reported TALD cases with onset of disease in 2015 was the highest number ever reported to the network with 1141 reported cases (Figure 3).

These cases were reported from 25 countries including 22 EU/EEA Member States and three non-EU/EEA countries which were Switzerland (30 cases), the USA ( 6 cases) and Australia ( 2 cases). Two-thirds (66.2\%) of all TALD cases were reported by the United Kingdom, Italy, France and the Netherlands. For the TALD cases, there was also a seasonal variation with two-thirds of the cases in 2015 emerging between June and October. There is also a similarity to the overall Legionnaires' disease sex distribution, as over two-thirds $(69 \%)$ of the reported TALD cases are male. Cases had a median age of 62 years and $82 \%$ of cases were aged 


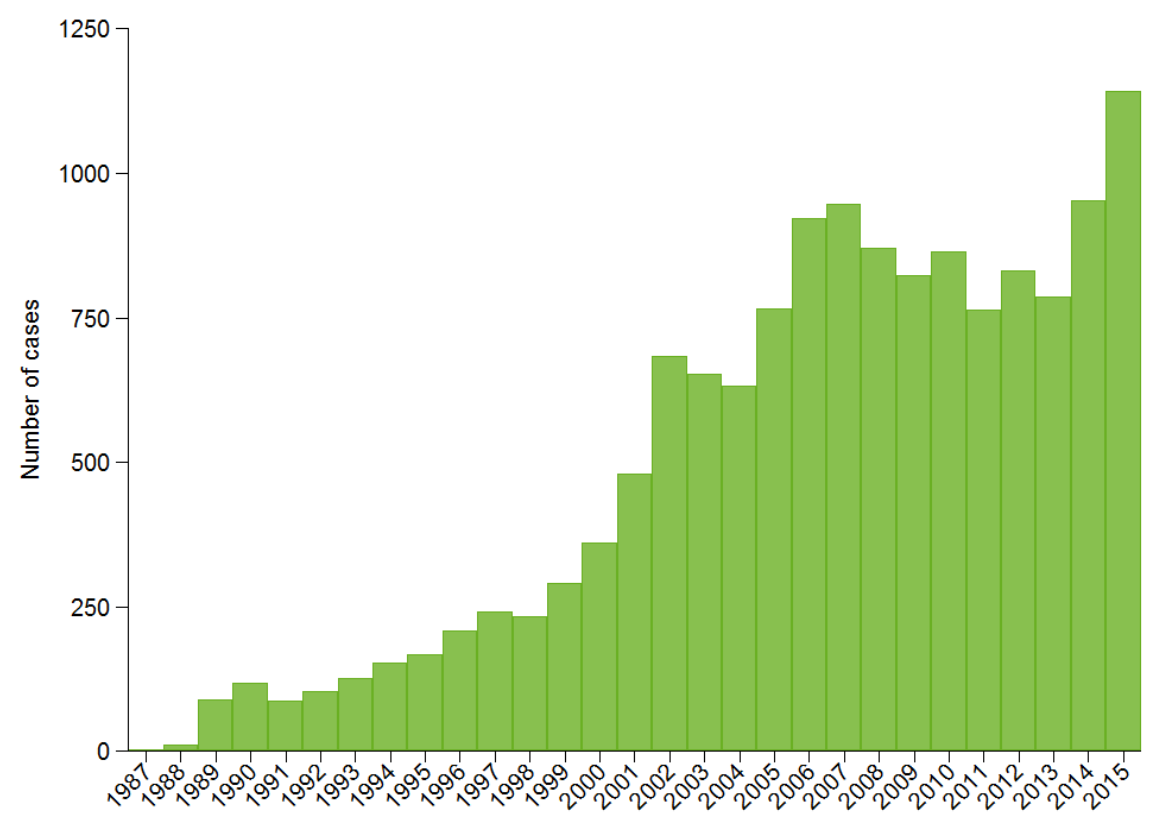

Figure 3. Number of travel-associated cases of Legionnaires' disease reported to ELDSNet, by year, 1987-2015. Source: https://ecdc.europa.eu/sites/portal/files/documents/Legionnares-diseaseeurope-2015.pdf

50 years or older. Around $90 \%$ of the TALD cases are diagnosed using a urinary antigen test. Since the TALD cases are reported in a very timely manner, the clinical outcome of infection is often known for only about half of the cases, and the case-fatality rate for those cases with a reported outcome is around 4\%. The TALD cases reported in 2015 were associated with a cumulative total of 1606 stays in various destinations around the world, and $76 \%$ were within the EU/EEA area (Figure 4).

In total, 167 new clusters were detected in 2015, and the majority were comprised of two cases. In $60 \%$ of the clusters, the first two reported cases were from different countries. Thus, most of these clusters would likely not have been detected without the ELDSNet network and EU 


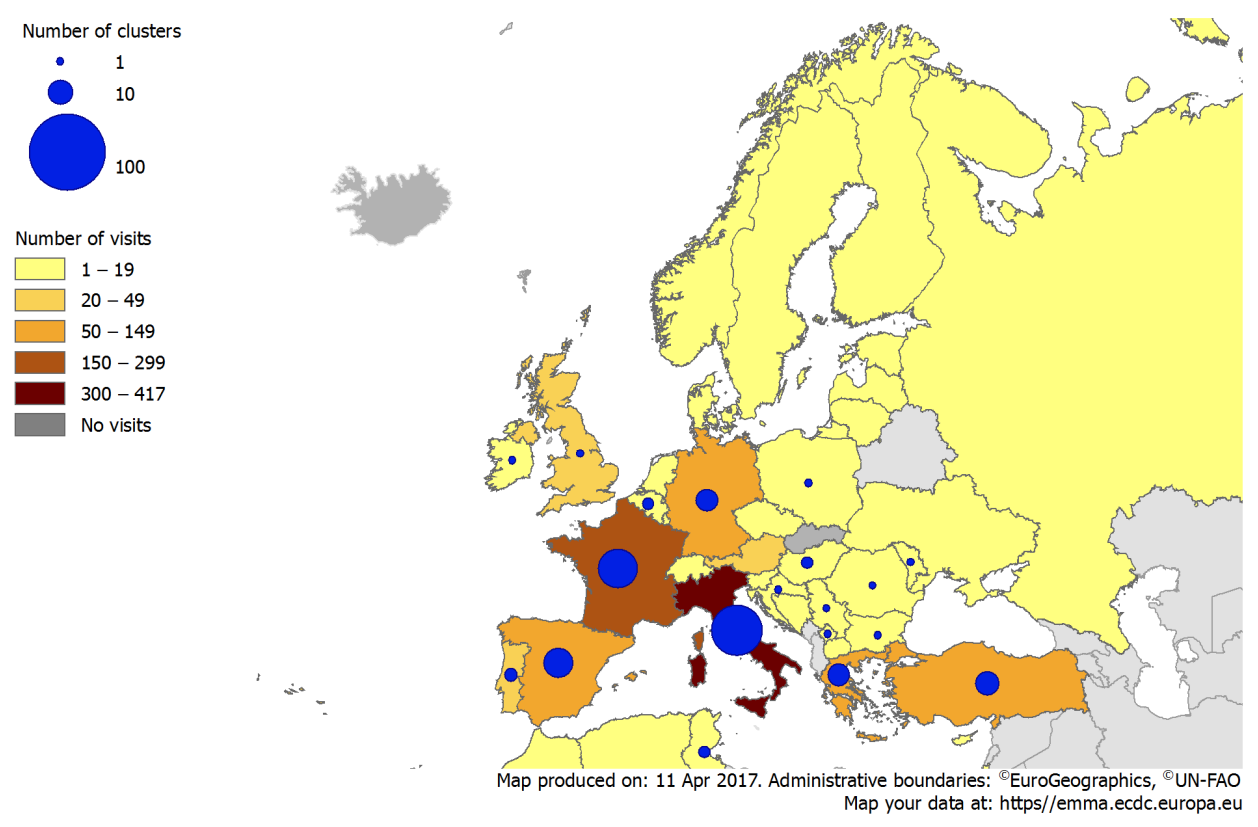

Figure 4. Number of accommodation site visits and clusters of travel-associated cases of Legionnaires' disease per destination country, EU/EEA and neighbouring countries, 2015. Source: https://ecdc.europa.eu/sites/portal/files/documents/Legionnares-disease-europe-2015.pdf

surveillance scheme. The number of TALD clusters at the subnational (NUTS2) is shown in Figure 5.

For all 167 clusters, the Forms A and B were received. Legionella bacteria were found in $60 \%$ of all cluster sites where environmental water sampling results were reported. Eight accommodation site names were published on the ECDC website, because the form B stated that satisfactory control measures had not been implemented in a timely manner.

\section{Conclusions}

Notification rates of Legionnaires' disease vary from 0.1 to 30.0 cases per million population in the EU/EEA countries. Four countries (France, Germany, Italy, and Spain) accounted for $69 \%$ of all notified cases in 2015, although their combined populations only represent approximately 

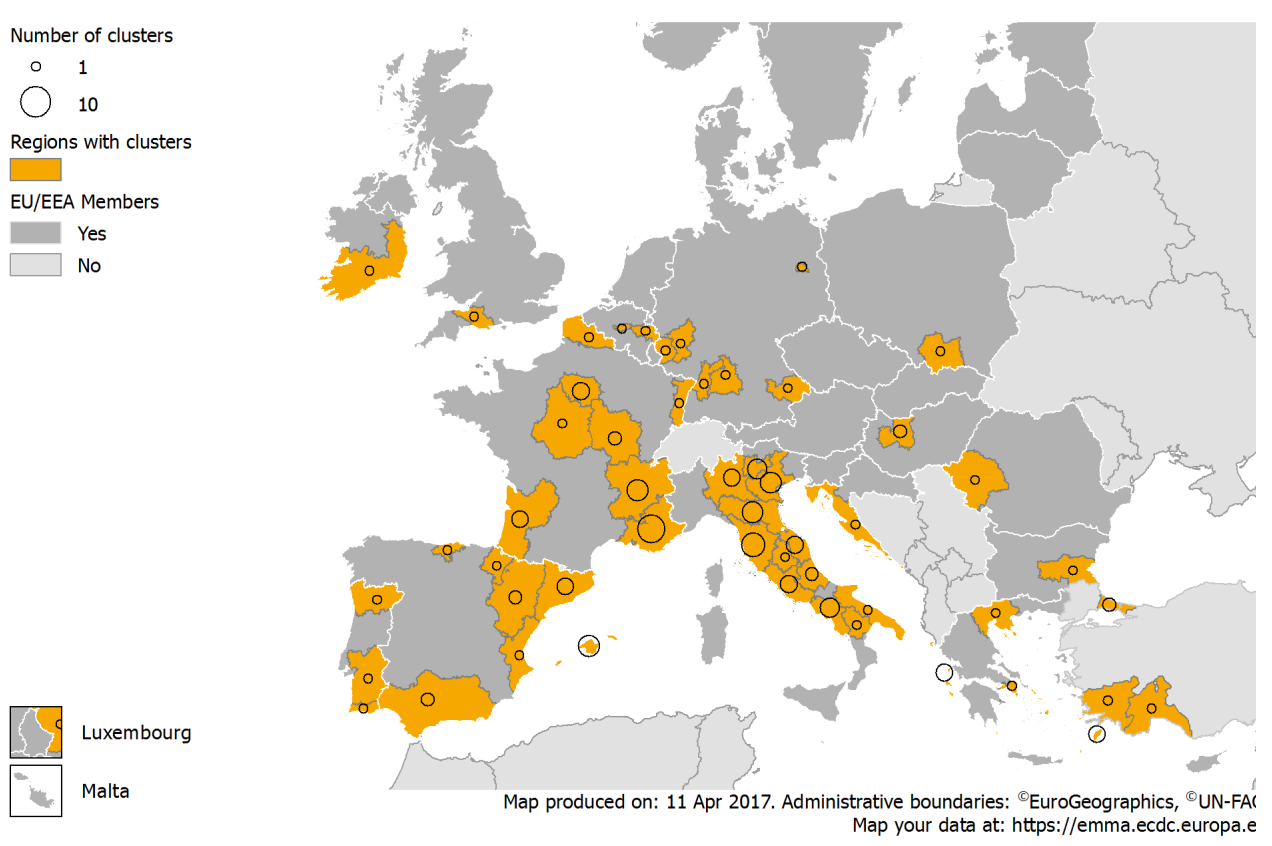

Figure 5. Number of clusters* of travel-associated Legionnaires' disease per destination area (NUTS2), EU/EEA, 2015 excluding complex clusters. Source: https://ecdc.europa.eu/sites/portal/ files/documents/Legionnares-disease-europe-2015.pdf

$50 \%$ of the EU/EEA population. Many countries have a notification rate below 0.5 cases per 100000 population which has been unchanged for the past five years and unlikely to reflect the true incidence of Legionnaires' disease. It is likely that the lower rates represent a considerable underestimation of the incidence which could be due to both underdiagnosis and underreporting. The European surveillance of the travel-associated cases as performed by ELDSNet is a very good example of how multi-country joint surveillance schemes can facilitate the implementation of preventive measures, and thereby reduce potential exposure sources for travellers at risk for Legionnaires' disease. 


\section{References}

ECDC Health Information Leaflet for managers of tourist accommodation on how to reduce the risk of Legionnaires' disease.

(2017). European Technical Guidelines for the Prevention, Control and Investigation, of Infections Caused by Legionella species.

(2012) Commission Implementing Decision 2012/506/EU of 8 August 2012 amending Decision 2002/253/EC laying down case definitions for reporting communicable diseases to the Community network under Decision No 2119/98/EC of the European Parliament and of the Council. 